\title{
VLA POLARIZATION OBSERVATION OF THE RADIO ARC AT 15 GHZ
}

\author{
M. Inoue ${ }^{1}$, E. Fomalont ${ }^{2}$, M. Tsuboi ${ }^{1}$, F. Yusef-Zadeh ${ }^{3}$, \\ M. Morris ${ }^{4}$, H. Tabara ${ }^{5}$, and T. Kato 5 \\ 1. Nobeyama Radio Observatory, Minamisaku, Nagano 384-13, Japan \\ 2. NRAO, Charlottesville, VA 22903, USA \\ 3. LASP, GSFC, and Northwestern University, USA \\ 4. UCLA, Los Angeles, CA 90024, USA \\ 5. Utsunomiya University, Utsunomiya, Tochigi 321, Japan
}

\begin{abstract}
Polarization measurements of the radio Arc were made with the VLA at $15 \mathrm{GHz}$. High frequency polarimetry made with high spatial resolution minimizes Faraday depolarization and reveals polarized filaments which correspond to the predominant filaments of the radio Arc. We notice a peculiar linear feature in the polarization map ("thorns") which suggests the presence of a second magnetic field system. The total intensity maps show no evidence for an interaction between the two field systems, so the thorns may be foreground magnetized structures. However, if the two magnetic field systems do interact, it would allow a model in which the acceleration of relativistic particles takes place at their intersection. The accelerated particles would flow toward both ends of the radio Arc, and account for the intrinsic polarization observed along the entire length of the system. Thermal electrons responsible for the Faraday depolarization occuring at longer wavelengths may be supplied by the interaction of the streams of relativistic particles with relatively dense, ambient thermal clouds.
\end{abstract}

\section{INTRODUCTION}

In addition to the complex distribution of molecular and ionized gas clouds, peculiar nonthermal features have been revealed in the galactic center region. Polarization of the radio filaments was reported by Yusef-Zadeh, Morris, and Chance (1984), and Inoue et al. (1984) found that the Faraday rotation toward the radio Arc was extremely large, >2500 rad $\mathrm{m}^{-2}$ (Tsuboi et al. 1986). Both groups suggested that the direction of the magnetic field is parallel to the collection of radio filaments composing the non-thermal portion of the Arc. In addition, two polarized plumes appear at the ends of the Arc and extend over a total of 1.6 degrees along a line perpendicular to the galactic plane (Tsuboi et al. 1985, 1986; Seiradakis et al. 1985). Furthermore, Tsuboi et al. (1986) determined the direction of the magnetic field, finding that the field lines are aligned with the radio filaments and the long axis of the plumes. The direction of the magnetic field along the plumes and along the filamentary structures in the radio Arc suggests not only that these structures are physically associated, but also that a large-scale magnetic field is oriented perpendicular to the galactic plane. On the other hand, except for the region near G0.16-0.15 (Yusef-Zadeh et al. 1986), little polarization has previously been seen on the radio Arc. This has been ascribed to Faraday depolarization by thermal gas which surrounds, or is intermixed with, the radio Arc (Tsuboi et al. 1986; Yusef-Zadeh et al. 1986). In spite of the ordered filamentary nature of the radio Arc, high-resolution polarization observations have shown a rather 
complex substructure (Yusef-Zadeh 1986; Yusef-Zadeh and Morris 1987). The polarized intensity at $5 \mathrm{GHz}$ is concentrated near G0.16-0.15, is very patchy , and does not follow the filamentary geometry of the total intensity. The non-uniform distribution of polarized intensity in G0.16-0.15 is attributed to a Faraday depolarizing medium which is roughly anticorrelated in position with the "helical segments" of radio emission surrounding the Arc (Yusef-Zadeh, Morris, and Chance 1984; Yusef-Zadeh 1986).

The amount of Faraday rotation, and consequently Faraday depolarization, is proportional to the square of the observing wavelength. Hence, high frequency polarization observations will provide the most direct information on the non-thermal characteristics of the filaments, i.e., on the structure of the magnetic field and on the acceleration mechanism of the radiating particles. We thus made $15-\mathrm{GHz}$ polarization observations of the radio Arc with the VLA, the results of which will be described here. Further details will be described elsewhere.

\section{OBSERVATIONS AND RESULTS}

The observations were made using the Very Large Array (VLA) in its hybrid C/D configuration with circularly polarized feeds at U band. The dual IF system was used with bandwidths of $50 \mathrm{MHz}$ and center frequencies of 14.565 and $15.015 \mathrm{GHz}$ in order to derive Faraday rotation. Twelve overlapping fields were observed mainly along the radio Arc with separations of about half of the primary HPBW $(=220 \operatorname{arcsec})$. In addition, the $\mathrm{B} / \mathrm{C}$ hybrid was also employed for observing the central two fields near G0.16-0.15.

Figure 1 shows the distribution of total $15-\mathrm{GHz}$ intensity in a mosaic of all twelve fields. Each field was CLEANED with a restored beam of 4.7 arcsec using the AIPS software system installed at NRO. In addition to a prominent filament located on the southwest side of the radio Arc, other filaments crossing the filamentary system can be seen, the most prominent of which is G0.18-0.04, known from early single-dish observations (e.g., Pauls et al. 1976). A second feature is located on the southeast portion of the radio Arc, extending north-south at R.A. $=17^{\mathrm{h}} 43^{\mathrm{m}} 35^{\mathrm{s}}$. Some other crossing features can be discerned at the northwest end of the radio Arc. These correspond to the eastern portion of the arched filaments (e.g., Yusef-Zadeh and Morris 1988).

The distribution of polarized intensity and polarization angle at $14.565 \mathrm{GHz}$ are shown in Figure 2. The polarized intensity is strong in the southeastern half of the radio Arc, where the compact polarized component appears at $10 \mathrm{GHz}$ (Inoue et al. 1984; Tsuboi et al. 1985, 1986; Seiradakis et al. 1985), and where complex structures appear in VLA maps at 5 and $15 \mathrm{GHz}$ (Yusef-Zadeh 1986; Yusef-Zadeh and Morris 1987). In this region, the predominant filament in the total intensity images coincides with the polarized filaments. Furthermore, we note two remarkable polarization properties: first, there are two linear features resembling "thorns" running across the polarized filament. It is unclear whether these should be interpreted as highly polarized filaments superimposed upon a lowpolarization background, or as filaments of depolarization superimposed upon a relatively smooth background of large polarization. A detail of the single $15-\mathrm{GHz}$ field containing the thorns is shown in figure 3. Although the thorns are prominent in polarized intensity images, there is no corresponding feature in the total intensity map (see Figure 4, which shows the same field with the polarized intensity image superimposed upon the total intensity contours). There are some other vertical polarized features parallel to the thorns, 

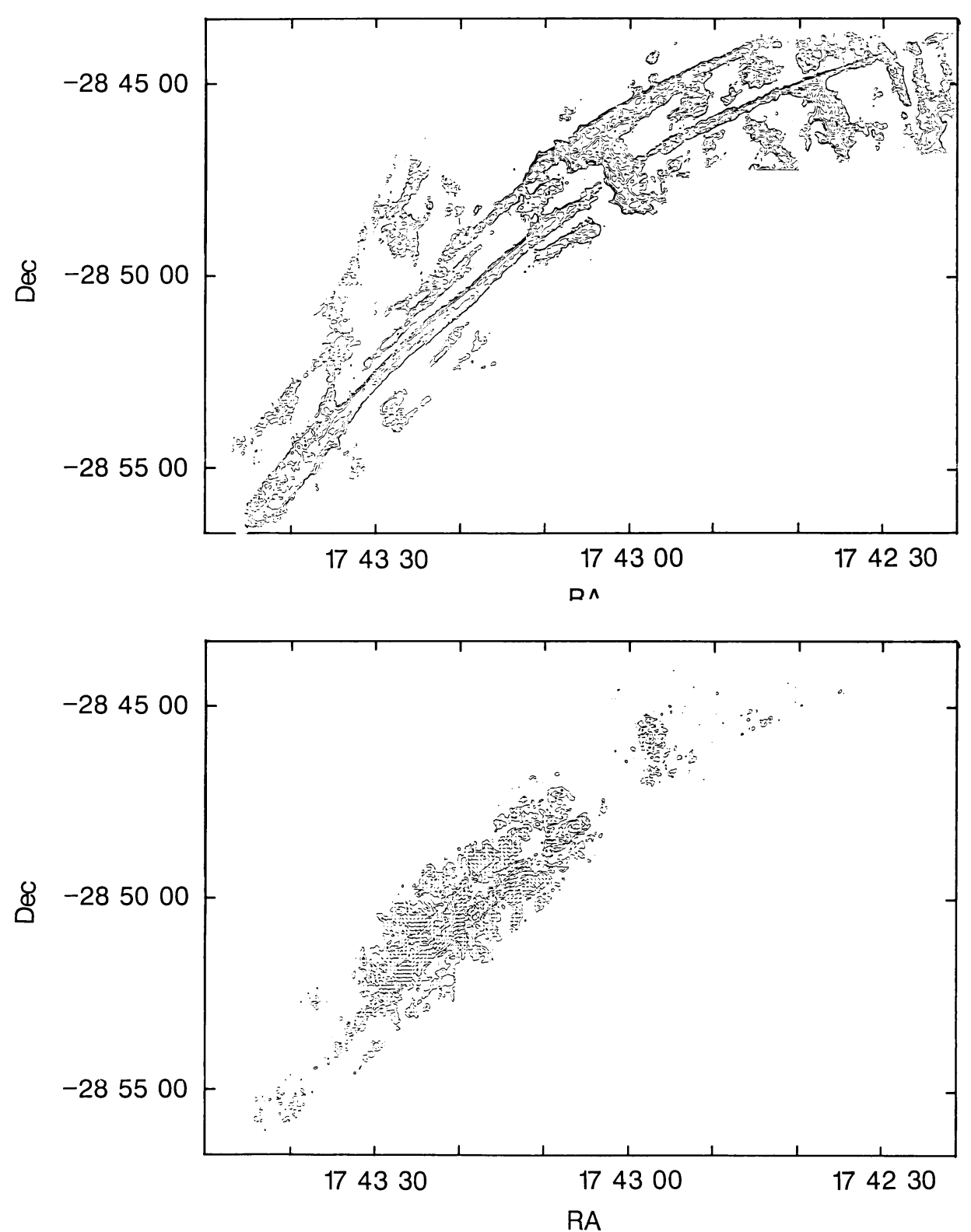

Figures 1 (top) and 2 (bottom): Contours of total and polarized intensity, respectively, in the linear filaments of the radio Arc. These figures result from a mosaic of the maps of the many overlapping fields (see text). The directions of the polarized electric field vectors are indicated by the line segments in figure 2. 
while all the structures in the total intensity run parallel to the predominant (polarized) filament.

The second remarkable characteristic is that the polarized intensity becomes weak where the crossing features in the total intensity, notably G0.18-0.04, cross the predominant linear filament. In addition, to the northwest of $\mathrm{G} 0.18-0.04$, polarized emission becomes very weak, although one can still trace the polarized filament. At the northwestern tip of the Arc, where the arched filaments cross the linear filaments, the polarization of the linear filaments disappears. Another vertical feature southeast of G0.18-0.04 (R.A. = $17^{\mathrm{h}} 43^{\mathrm{m}} 10^{\mathrm{S}}$, see Yusef-Zadeh, Morris, and van Gorkom, in these proceedings) also shows a depression of the polarized intensity along the filament.

\section{DISCUSSION}

Whereas the linear filaments represent a magnetic field structure which is poloidal, the linear polarized thorn features crossing them suggest the presence of a second magnetic field system. The question arises whether the two systems are interacting. Indeed, strong polarization around the thorns might imply that acceleration of the relativistic particles takes place as a result of the interaction of the magnetic field systems, with consequent field line annihilation. Then, the strong polarization in the vicinity of the thorns might be explained in terms of a concentration of newly generated relativistic electrons. These electrons would propagate toward both ends of the Arc along the linear filaments. One might suppose that, as they stream toward the ends, the relativistic electrons interact with the thermal crossing features and generate an admixture of thermal electrons within the flow. The entrained thermal electrons would then cause Faraday depolarization of the synchrotron emission from the relativistic electrons, so that the polarization disappears in the vicinity of the thermal crossing features.

On the other hand, the complete absence of a total intensity counterpart to the thorns could mean that no such interaction is occuring, and that the thorns represent a magnetized, foreground structure. The positional coincidence of the thorns with the "helical segments" suggests that the thorns might be identified with a thermal sheath surrounding the Arc, as described by Yusef-Zadeh et al. (1986). In this case, the high polarization in the vicinity of the thorns corresponds to a region of uncharacteristic magnetic field uniformity in the otherwise chaotic and therefore depolarizing medium of the sheath. This latter hypothesis does not negate the possibility that the depolarizing medium is intermixed with the region of polarized emission. If the thorns are to be interpreted as depolarizing features, then they are presumably outside, and in front of, the polarized region.

\section{REFERENCES}

Inoue, M., Takahashi, T., Tabara, H., Kato, T., and Tsuboi, M. 1984, Publ. Astron. Soc. Jpn., 36, 633.

Pauls, T. Downes, D., Mezger, P.G., and Churchwell, E. 1976, Astron. Ap., 46, 407.

Seiradakis, J.H., Lasenby, A.N., Yusef-Zadeh, F., Wielebinski, R., and Klein, U. 1985, Nature, 317, 679.

Tsuboi, M., Inoue, M., Handa, T., Tabara, H., and Kato, T. 1985, Publ. Astron. Soc. Jpn., 37, 359. 
Tsuboi, M. Inoue, M., Handa, T., Tabara, H., Kato, T., Sofue, Y., and Kaifu, N. 1986, Astron. J., 92, 818.

Yusef-Zadeh, F. 1986, PhD Thesis, Columbia University.

Yusef-Zadeh, F. and Morris, M. 1987, Astrophys. J., 322, 721. 1988, Astrophys. J., 329, 729.

Yusef-Zadeh, F., Morris, M., Slee, O.B., and Nelson, G.J. 1986, Astrophys. J., 310, 689.

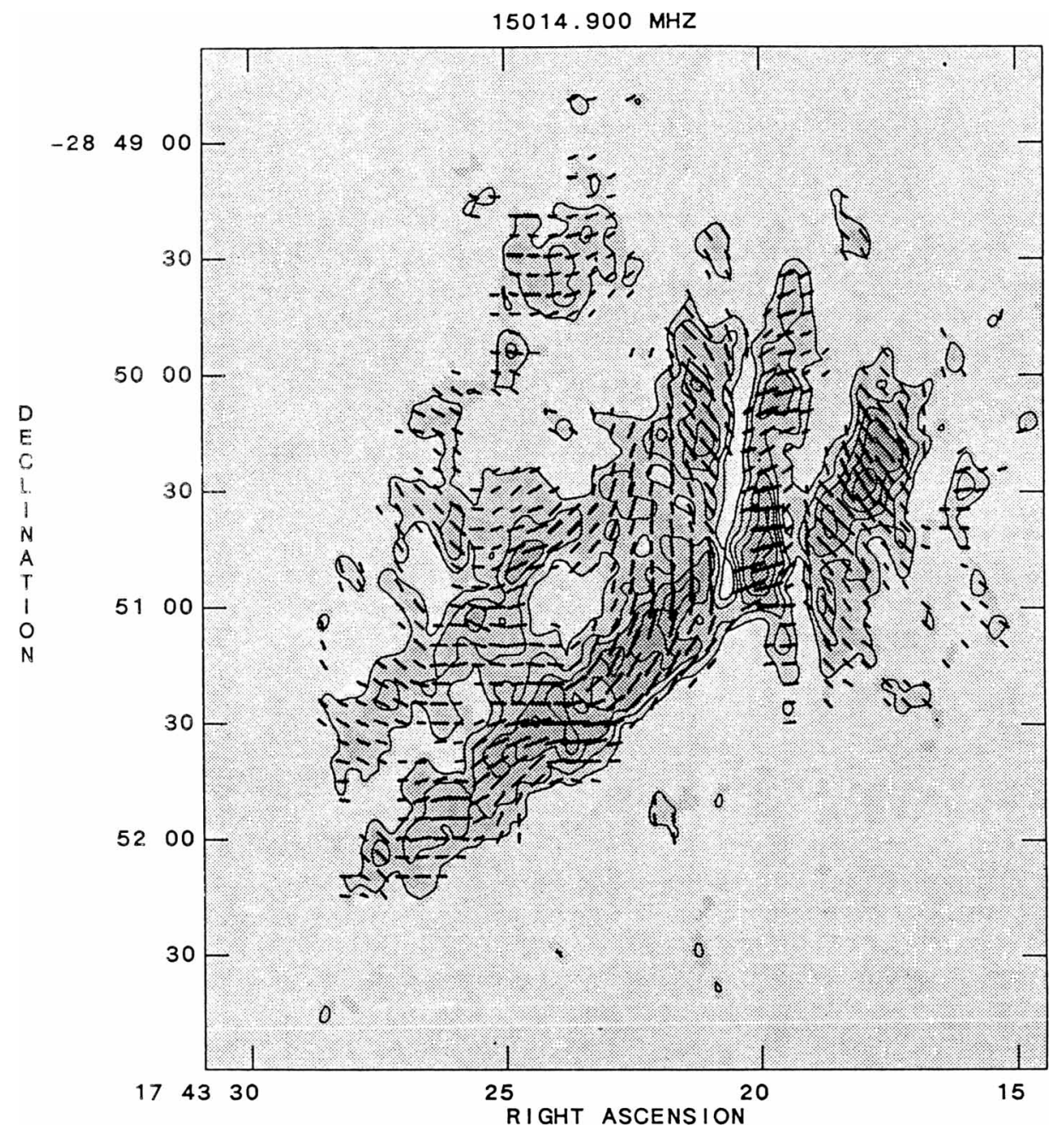

Figure 3: Polarized intensity contour map of one field at $15.015 \mathrm{GHz}$, with electric vectors superimposed. This map is not corrected for the response of the primary beam. Contour levels are $2.5,4,5.5,7$, ..., $14.5 \mathrm{mJy} /$ beam area. 

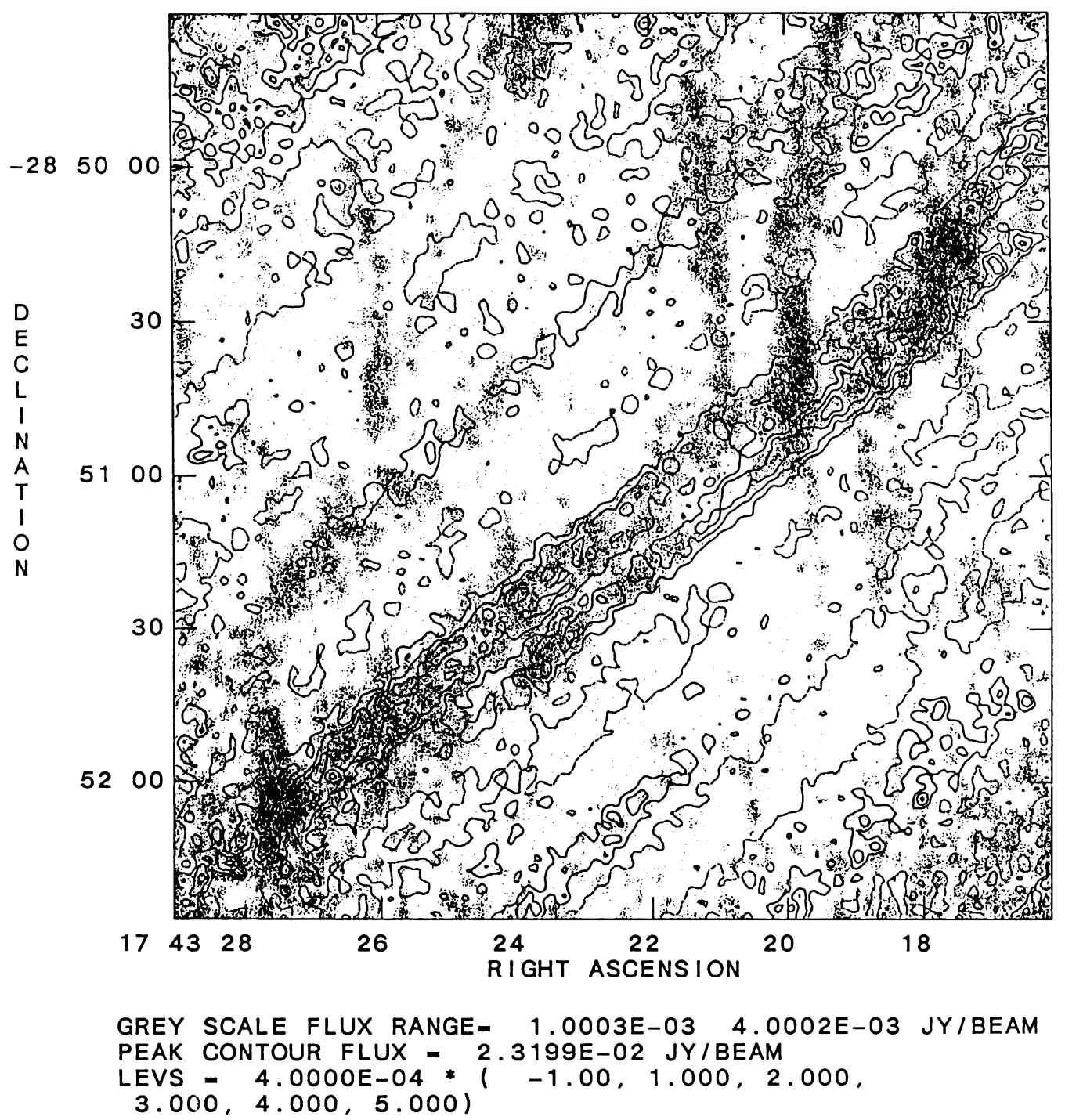

Figure 4: Superposition of the polarized intensity image at $15 \mathrm{GHz}$ (gray scale) upon the total intensity (contours) in the region that includes the "thorns". 